\title{
Lidil
}

Revue de linguistique et de didactique des langues

56 | 2017

Grammaires et littéracies

\section{La cohérence textuelle et la grammaire de la phrase dans les productions écrites de doctorants}

Textual Coherence and Sentence Grammar in Material Written by Doctoral Students

Ève Lejot

\section{OpenEdition}

\section{Journals}

Édition électronique

URL : http://journals.openedition.org/lidil/4785

DOI : 10.4000/lidil.4785

ISSN : 1960-6052

Éditeur

UGA Éditions/Université Grenoble Alpes

Édition imprimée

ISBN : 978-2-37747-024-2

ISSN : 1146-6480

\section{Référence électronique}

Ėve Lejot, « La cohérence textuelle et la grammaire de la phrase dans les productions écrites de doctorants », Lidil [En ligne], 56 | 2017, mis en ligne le 01 novembre 2017, consulté le 02 mai 2019. URL : http://journals.openedition.org/lidil/4785 ; DOI : 10.4000/lidil.4785

Ce document a été généré automatiquement le 2 mai 2019.

(C) Lidil 


\title{
La cohérence textuelle et la grammaire de la phrase dans les productions écrites de doctorants
}

\author{
Textual Coherence and Sentence Grammar in Material Written by Doctoral
}

Students

Ève Lejot

\section{Introduction}

1 Les attentes et le mode de fonctionnement des institutions universitaires sont peu verbalisés, notamment en termes de savoirs à l'écrit (De Schepper, 2010, p. 93), et les étudiants doivent trouver leur voie pour communiquer efficacement. L'objectif de la présente recherche est de créer un pont entre la grammaire de la phrase et la cohérence textuelle dans les écrits académiques auprès de doctorants natifs et non natifs dans le domaine de la littéracie universitaire.

2 Notre approche didactique consiste à passer par une unité connue, la phrase, pour accompagner les doctorants relativement au concept de pertinence de l'énoncé.

Lorsqu'on conçoit la grammaire en termes de "possible ou grammatical» selon Cuq (1996), on se retrouve dans une appréciation binaire : correct ou non. De plus, selon le concept de compétence de Chomsky (1965), un énoncé peut aussi être agrammatical et acceptable. Si on pense la grammaire non en termes de " conformité » mais en termes de «structures» de l'énoncé dans ses dimensions syntaxiques et morphologiques pour reprendre les termes du CECRL (2000, p. 89), qui définit la grammaire comme « l'ensemble des principes qui régissent la combinaison d'éléments en chaines significatives marquées et définies ", alors on peut penser le cours à un niveau avancé ${ }^{1}$ non plus en termes de justesse grammaticale, mais en termes de capacité à manipuler les principes et structures grammaticales pour mieux véhiculer les idées, mettre en avant ce qui doit l'être, relier, opposer ou nuancer. Cette approche permet aux doctorants de se préparer aux révisions 
auxquelles les textes écrits académiques sont en principe soumis (Becker, 1986). Notre constat est le suivant: ils «copient-collent» des extraits de textes travaillés ici et là et n'ont pas toujours une vision d'ensemble suffisante pour que leur texte soit cohérent.

Dans la présente contribution, nous souhaitons proposer une écriture "théorisante " (Laborde-Milaa, Boch \& Reuter, 2004) afin que les étudiants puissent interpréter la théorie énonciative, puis phraséologique et, enfin, grammaticale dans leur contexte rédactionnel, appliqué au genre du doctorat et dans un contexte disciplinaire précis.

5 À l'université du Luxembourg, les doctorants en droit souhaitent suivre un cours sur les écrits académiques qui leur soit spécifiquement destiné. Nous offrons donc systématiquement deux cours distincts pour les doctorants en sciences humaines et les doctorants en droit. Nous avons, par suite, cherché à savoir si, pour des thématiques précises telle que la cohérence textuelle, il existe des différences notables dans les productions écrites de ces deux groupes. En conséquence, nous avons mené cette étude en parallèle auprès de deux groupes : les doctorants en sciences humaines d'un côté et ceux en droit de l'autre. La question qui se pose ici est de savoir ce qui est " enseignable " selon les disciplines (Pollet, 2004) pour travailler sur la qualité des écrits de doctorants natifs et non natifs dans un même groupe. Nous nous intéresserons à la dimension grammaticale, consciente ou non, dans le travail des doctorants sur la cohérence textuelle de deux paragraphes extraits de leur propre recherche et nous efforcerons de répondre aux questions suivantes: observe-t-on une différence entre le premier texte apporté en cours et sa nouvelle version d'un point de vue grammatical? Quelle est la réaction des doctorants face à cette approche ? Observe-t-on une différence selon que les doctorants sont francophones natifs ou non, qu'ils sont doctorants en sciences humaines ou en droit?

Nous formulons l'hypothèse que la compétence grammaticale de ces doctorants est insuffisamment exploitée et qu'une nouvelle sensibilisation à la construction de la phrase pourrait permettre de façonner leurs écrits comme le propose la définition du CECRL.

7 Dans la première partie de cet article, nous présenterons la cohérence comme un levier au cœur de la réécriture. Dans la seconde partie, nous analyserons les modifications que les doctorants ont apportées à leurs écrits et les évaluations du cours réalisées par les doctorants. Enfin, nous interrogerons la pertinence de cette approche par le retour à la phrase.

\section{La cohérence : levier pour la réécriture}

Le processus d'écriture exige des phases d'engagement face à la page blanche afin de modeler le fil de la pensée. Ce processus a une fonction cognitive de l'écrit (Goody, 1979) et s'opère en plusieurs étapes (Becker, 1986), par touches et couches successives. Réécrire, au sens de réviser son texte, c'est non seulement procéder à des révisions locales qui n'engagent ni la macro ni la superstructure du texte, mais aussi accepter de retravailler la matière et de se confronter à son texte pour le réorganiser et en garder l'essence.

\subsection{La révision}

9 La révision doit prendre en considération le lecteur. Ce facteur est d'autant plus important qu'il s'agit de productions académiques qui font l'objet d'évaluations. Le 
lecteur doit être intéressé, captivé au sens propre et au sens figuré. La communication doit faire preuve de cohérence, à l'écrit comme à l'oral. Au regard des nombreuses recherches (Beaugrande \& Dressler, 1981; Charolles, 1978; Hobbs, 1983; Halliday \& Hasan, 1976) réalisées à ce jour sur la cohérence textuelle, Gagnon (2015) reprend les études menées sur la cohérence et considère que cette dernière « repose notamment sur la reprise d'éléments d'information déjà introduits et l'introduction d'informations nouvelles ». Le texte est un « casse-tête » dans lequel « chaque pièce (énoncé) a sa raison d'être » (Gagnon, 2003, p. 62).

10 L'ordre des arguments et les outils de transition constituent les éléments-clés de la construction d'un texte. Les énoncés ou phrases doivent répondre à deux principes :1) la pertinence ou raison d'être de l'énoncé ; 2) l'arrimage ou le lien des énoncés entre eux (Gagnon 1998; Gagnon, 2003), essentiel pour « façonner l'image globale » (Gagnon, 2003, p. 62).

11 La cohérence textuelle correspond par ailleurs à l'alliance entre un message clairement contextualisé grâce à des énoncés antérieurs et à une relation vraisemblable entre ces derniers, et ce, « au prix d'un effort minimal de la part du lecteur » (Gagnon, 2015, p. 171). Le concept de cohérence textuelle est ici pour nous un levier théorique qui permet aux doctorants de revisiter la syntaxe de leur phrase et donc de repenser l'opérationnalité de la phrase simple et de la phrase complexe dans leurs textes.

12 Le sujet peut sembler "simpliste » à première vue, voire «indigne" de doctorants. Pourtant, des analyses de commentaires entre pairs révèlent des lacunes dans la construction de l'argumentation, laissant le lecteur démuni face aux informations présentées par les doctorants, ne lui donnant pas l'envie nécessaire à la poursuite de sa lecture. Afin de permettre aux étudiants de construire leur texte autour de leurs idées et de s'octroyer la confiance et la sensibilité au potentiel gain cognitif du lecteur, nous avons envisagé de leur proposer quelques clés de rédaction permettant d'obtenir une véritable cohérence textuelle. Ainsi, à partir de l'organisation de phrases ou, plutôt d'énoncés, nous avons souhaité les amener à réfléchir sur l'organisation plus globale de leurs idées en contexte et sur la pertinence de leurs énoncés. Partant de leurs énoncés, les étudiants sont invités à construire des paragraphes cohérents.

13 Toutefois, n'étant pas une enseignante spécialiste de leur discipline, nous ne nous concentrerons pas sur la cohérence de leur texte, sur le choix des idées ni sur la manière de les articuler, d'un point de vue logique, les unes aux autres, mais nous nous intéresserons à la cohésion textuelle, puisqu'elle est une condition nécessaire à la cohérence textuelle.

\subsection{Conception et déroulement de l'atelier d'écriture}

14 La partie théorique du cours ne durant que $1 \mathrm{~h} 30$, nous avons avant tout sensibilisé les étudiants aux concepts de pertinence (Sperber \& Wilson, 1989) et d'arrimage (Gagnon, 2015). Nous avons concentré notre enseignement sur un niveau métalinguistique pour rappeler aux doctorants que leurs écrits ont des destinataires qui attendent un gain cognitif à moindre effort (Moeschler \& Reboul, 1994, p. 92). La question de l'arrimage a été l'occasion de refaire un point sur les connecteurs explicites (conjonction de coordination, adverbes, etc.) et les connecteurs implicites (anaphores, temps verbaux, pronoms, etc.) entre les énoncés. Dans une seconde étape, nous sommes passés à la 
réalisation d'exercices de repérage d'énoncés cohérents ou non au sein de paragraphes propres à leurs domaines de recherche.

\subsubsection{Création de matériel de cours à partir de Scientext} Loiseau, 2013) ou de textes de certains doctorants, les objectifs du cours proposé sont non seulement devenus plus visibles, mais aussi plus immédiats, pour envisager les possibilités de progression des écrits que l'on peut atteindre en réfléchissant en termes de cohérence textuelle.

La base de données Scientext regroupe des textes scientifiques (thèses, HDR et articles scientifiques) ; elle est dotée d'un moteur de recherche et permet donc de retrouver des textes liés à un domaine d'études particulier. Tout l'intérêt de Scientext est de permettre une recherche de textes en lien avec les problématiques des doctorants. Le moteur de recherche en fait un outil pratique et riche en contenu. Nous avons donc envisagé de sélectionner dans la base de données des textes authentiques pour construire un cours adapté aux doctorants des deux groupes que nous voulions sensibiliser à la cohérence textuelle. C'est ainsi que, dans un premier temps, nous avons conçu des exercices à partir de Scientext.

17 Cependant, la richesse de Scientext a considérablement ralenti l'élaboration du cours : nous avons passé beaucoup trop de temps en recherches, à trier des données trop nombreuses et, finalement, des textes proches des problématiques des étudiants, mais peut-être pas suffisamment.

\subsubsection{La relecture entre pairs}

Les activités préparées en amont du cours ont pour but de mettre les doctorants face à leur double posture de scripteur et de lecteur. Les étudiants participent à des sessions de relecture entre pairs depuis deux semestres déjà et ils passent donc régulièrement du statut de scripteur à celui de lecteur.

\subsubsection{Deux groupes, deux mises en route distinctes}

19 À l'issue du cours avec le premier groupe - les doctorants en sciences humaines -, nous avons fait plusieurs observations qui nous ont amenée à repenser ce cours. La première remarque que nous pouvons formuler est que la variété des problématiques au sein d'un même groupe rend probablement le travail entre pairs moins riche, et la cohésion moins forte. Ensuite, notre préparation en amont avec Scientext a permis aux étudiants de travailler sur des textes abordant des sujets proches des leurs, mais tout de même trop éloignés pour que cette attention soit visible et retenue.

Les cours étant programmés à une semaine d'intervalle, le second groupe d'étudiants - les doctorants en droit - a pu bénéficier d'une réadaptation. Nous avons choisi de faire travailler ces étudiants sur leurs propres textes, enrichis d'incohérences afin de mieux coller à nos démonstrations. Ce choix a généré un résultat satisfaisant : l'utilisation des problématiques similaires au sein d'un même groupe a probablement rendu le travail entre pairs plus riche, et la cohésion du groupe plus forte. En outre, les étudiants ont apprécié de travailler sur des textes connus, les leurs ou ceux de leurs pairs. 
21 Notre premier objectif, à l'issue de la séance consacrée à la cohérence textuelle, était que les étudiants soient capables de comprendre que la cohérence de leurs idées et celle de leur syntaxe étaient intimement liées (l'une ne va pas sans l'autre). Notre zone d'impact se situant autour de la cohérence de leurs textes, notre second objectif était qu'ils soient capables de prendre de la hauteur au moment de rédiger leurs énoncés, afin d'adopter le point de vue du lecteur auquel leurs habitudes de relectures entre pairs les avaient préparés (sans pour autant que cette posture soit interrogée consciemment jusqu'ici).

\section{L'analyse des modifications portées à un paragraphe}

Pour les catégories d'analyse, nous nous sommes concentrée sur les changements apportés entre le paragraphe original et la version retravaillée. Les doctorants sont intervenus sur leur texte de deux manières : dans le texte lui-même et en le commentant. Les commentaires leur ont permis de développer un regard métadiscursif sur leurs propres écrits. Pour définir les catégories d'analyse, nous nous sommes inspirée des matrices développées pour les activités de relecture entre pairs.

\subsection{Dimensions locale et globale}

23 Un commentaire peut porter sur la « microstructure » et/ou la " macrostructure » (Min, 2006, p. 126) ou, selon les termes de Cho et McArthur (2010, p. 333), sur le «surface level change ", le "micro-levelmeaning", le "macro-levelmeaning». Ces changements de niveau portent sur des erreurs grammaticales ou morphologiques et on retrouve ici, eu égard à la micro et à la macrostructure, respectivement les caractéristiques liées aux informations apportées par les texte et celles fournies par les connaissances préalables de l'individu qui lit sur le sujet (Van Dijk \& Kintsch, 1983). Ces connaissances préalables proviennent d'expériences antérieures ou de textes rencontrés précédemment avec des structures de base similaire au texte lu. Dans les comparaisons de textes académiques français et américains, Donahue (2008) travaille sur la cohérence des textes à trois niveaux d'analyse : la macro-cohérence qui porte sur la mise en page et la division en paragraphes, la méso-cohérence qui traite de l'intégration des références théoriques par la paraphrase et la citation et, enfin, la micro-cohérence qui répertorie les connecteurs et les déictiques. Cote (2013), quant à lui, a analysé les répercussions de l'attitude des étudiants sur la relecture entre pairs. Il a défini sa grille d'analyse à partir de dimensions «locale» ou " globale» (p. 122). La dimension globale porte sur le contenu du texte et enrichit significativement le propos, alors que la dimension locale intervient sur des ajustements relatifs à la forme du texte et apporte des corrections grammaticales, lexicales et/ou orthographiques.

Contrairement à l'ensemble des recherches citées précédemment, nous ne travaillons pas ici avec les doctorants sur un texte entier, mais sur un paragraphe pour en dénouer les mécanismes. Nous avons choisi cette échelle d'intervention afin de prendre de la distance par rapport à la dimension globale d'un texte académique et nous concentrer ainsi sur l'unité " phrase/énoncé ». Ce travail à un niveau micro offre l'opportunité aux doctorants de réapprendre à formuler leurs idées en repensant la construction de la phrase. Notre corpus est composé de 22 paragraphes commentés, soit 112 commentaires et 
changements au total, ainsi que de 18 questionnaires d'évaluation sur la séquence par les doctorants.

Pour notre analyse, nous avons choisi d'utiliser une matrice connue des doctorants, que nous leur avions présentée le semestre précédent dans le cadre de la relecture entre pairs (Lejot, 2016). Cette matrice comporte deux parties, « locale » et " globale », ainsi que trois sous-catégories : évaluation, clarification et altération dans le sens de modification.

Tableau 1. - Catégories de relecture entre pairs.

\begin{tabular}{|c|c|c|c|}
\hline Catégorie & Global & Local & \\
\hline Évaluation & Ce n'est pas un énoncé de thèse clair. & Ce mot n'a pas de sens. & \\
\hline Clarification & $\begin{array}{l}\text { Pourrais-tu expliquer ton idée } \\
\text { plus en détail? }\end{array}$ & $\begin{array}{l}\text { Que veux-tu dire avec } \\
\text { cela? }\end{array}$ & \\
\hline \multirow{4}{*}{ Altération } & \multirow{4}{*}{ Inverse tes arguments. } & \multirow{4}{*}{$\begin{array}{l}\text { Change « } \mathrm{x} » \text { avec « } \mathrm{y} » \text {. } \\
\text { Grammaire } \\
\text { Orthographe } \\
\text { Expressions } \\
\text { Structure }\end{array}$} & Ajout \\
\hline & & & Suppression \\
\hline & & & Remplacement \\
\hline & & & Inversion \\
\hline
\end{tabular}

Les altérations à un niveau global portent sur l'ordre d'exposition des arguments et, au niveau local, se focalisent sur des ajouts et des substitutions - que nous verrons plus en détail au cours de l'analyse. Les évaluations n'ont pas de buts correctifs, mais permettent à l'auteur de formuler ses impressions en tant que relecteur de son propre texte. Les clarifications sont souvent formulées sous forme de questions qui concernent l'explicitation du propos en demandant soit la réécriture d'une partie obscure du texte, soit des compléments d'information.

Nous allons voir que cette nomenclature n'a finalement pas su refléter dans le détail le parcours réflexif des doctorants sur leurs propres textes et que nous avons dû l'affiner à l'aide de sous-catégories au fil de nos analyses.

\subsection{Modifications des doctorants en droit et en sciences humaines}

Un premier résultat de l'ensemble des analyses qui répond à une des questions de recherche a trait à la différence sur les modifications apportées entre les doctorants en droit et ceux en sciences humaines ou encore entre les doctorants de langue maternelle française et les allophones. Il ne nous semble pas judicieux de distinguer expressément les doctorants en droit et en sciences humaines pour l'ensemble de l'analyse, car la proportion des répartitions des corrections par catégorie est sensiblement la même. Nous n'avons pas observé de différences notables entre les doctorants de langue maternelle française et les autres, ce qui est cohérent dans la mesure où les doctorants ont un niveau minimum $\mathrm{C} 1$ et ne doivent donc plus rencontrer de problèmes de grammaire de FLE.

Les premiers résultats d'analyse sont présentés dans le tableau 2. 
Tableau 2. - Types de modifications effectuées pour chaque catégorie de doctorants.

\begin{tabular}{|l|l|l|l|}
\hline & Droit & Sciences humaines & Total \\
\hline Altération & 54 & 45 & 99 \\
\hline Clarification & 6 & 4 & 10 \\
\hline Évaluation & 2 & 0 & 2 \\
\hline Total & $\mathbf{6 2}$ & $\mathbf{4 9}$ & $\mathbf{1 1 1}$ \\
\hline
\end{tabular}

Ces chiffres correspondent aux pourcentages suivants :

- $89 \%$ d'altérations ${ }^{2}$;

- $9 \%$ de clarifications :

"J'ai échangé [P3] avec [P4] pour donner une suite plus logique au texte. En [P2], l'ajout de "avant et même après l'unification" relie mieux [P1] et [P2] et donne de ce fait plus de pertinence à [P2]. L'ajout de "de ce fait" en [P3] montre que [P3] est une addition de même qu'une justification. »

"J'ai ajouté dans [P1] la raison pourquoi cet acquittement était obligatoire. L'ajout de [P3] explique [P2] et donne plus de pertinence au paragraphe. »

- $2 \%$ d'évaluations :

«Je sais c'est un peu lourd, mais je veux caser l'intitulé de la directive dans le corps du texte. Si jamais cela ne va pas je peux toujours le mettre en bas de la page. C'est un paragraphe rédigé aujourd'hui et je n'ai pas encore de distance par rapport au style. »

[Énoncé 6 avant] La Commission quant à elle, se fait l'écho d'une telle ambition à travers la proposition de directive relative à la lutte contre le terrorisme et remplaçant la décision-cadre 2002/475/JAI du Conseil relative à la lutte contre le terrorisme.

31 Comme les doctorants se corrigent eux-mêmes, ils ne sont pas dans une démarche d'évaluation ou de clarification, mais procèdent directement à des altérations de leurs écrits pour voir une amélioration. Ils ne sont pas dans la recherche de dialogue comme lors d'une relecture entre pairs. De plus, le travail sur un unique paragraphe est naturellement un facteur perturbant puisqu'il est dénué de contexte. Les doctorants interviennent par conséquent à $97 \%$ à un niveau local.

Tableau 3. - Modifications globales et locales effectuées par chaque catégorie de doctorants.

\begin{tabular}{|l|l|l|l|}
\hline & Droit & Sciences humaines & Total \\
\hline Global & 2 & 1 & 3 \\
\hline Local & 61 & 49 & 110 \\
\hline Total & 63 & 50 & 113 \\
\hline
\end{tabular}


Ils perdent l'ensemble du texte de vue et se concentrent sur les détails. Ce résultat peut s'expliquer par le fait que les doctorants travaillent sur un paragraphe qui est décontextualisé.

\subsection{Nature des altérations}

Comme nous venons de le voir, la plupart des changements opérés sont des altérations à un niveau local. Parmi ces nombreux changements, les doctorants ont ré-agencé les énoncés et ont procédé à des substitutions lexicales, des ajouts de mots au sein d'un même énoncé. Nous avons répertorié ces altérations en plusieurs catégories :

Tableau 3. - Nature des altérations effectuées par chaque catégorie de doctorants.

\begin{tabular}{|l|l|l|l|}
\hline & Droit & Sciences humaines & Total \\
\hline Métaréflexion & 2 & 1 & 10 \\
\hline Modification au sein d'un énoncé & 30 & 21 & 51 \\
\hline Réorganisation de l'ordre des énoncés & 6 & 9 & 15 \\
\hline Réorganisation de deux énoncés en un seul & 4 & 2 & 6 \\
\hline Réorganisation d'un énoncé en plusieurs & 12 & 12 & 24 \\
\hline Total & $\mathbf{5 4}$ & 55 & $\mathbf{9 9}$ \\
\hline
\end{tabular}

Parmi ces catégories, on observe trois tendances principales. La principale modification se fait au niveau de l'énoncé (et non du paragraphe).

Le deuxième niveau de modification concerne le paragraphe. En réorganisant leurs énoncés au sein du paragraphe, les doctorants prennent davantage de distance par rapport à leur travail. Toutefois, ce deuxième niveau semble être plus facile après une première réorganisation au sein de l'énoncé. Le troisième niveau de modification concerne la métaréflexion. Les étudiants s'interrogent (et interrogent ainsi leurs pairs) quant à la forme à donner à leur énoncé modifié.

\subsubsection{La première modification : au sein de l'énoncé}

37 Le travail de reprise au sein de l'énoncé correspond à $48 \%$ des modifications et se concrétise principalement par 3 actions :

- la substitution d'un connecteur à un autre :

[Énoncé 2 avant] Ainsi, nous percevons la famille comme un lieu d'échanges linguistiques dynamiques sujet aux pressions linguistiques externes et, dans lequel chaque personne joue un rôle essentiel dans le maintien de l'équilibre communicationnel.

[Énoncé 2 après] Néanmoins, nous percevons la famille comme un lieu d'échange linguistique dynamique dans lequel chaque individu joue un rôle essentiel dans le maintien de l'équilibre communicationnel de cette entité. 
[Énoncé 2 avant] Par ailleurs un questionnaire réalisé en 1812 à l'initiative du secrétaire général du département, Jean-Baptiste Christiani, met l'accent sur l'importance de l'expérience dans le processus de cooptation ou de désignation des anciennes accoucheuses.

[Énoncé 2 après] De plus, un questionnaire réalisé en 1812 à l'initiative du secrétaire général du département, Jean-Baptiste Christiani, révèle l'importance de l'expérience dans le processus de cooptation ou de désignation des anciennes accoucheuses.

[Énoncé 2 avant] Pour ce qui est du contrôle des normes infra-légales, le juge ordinaire veille à la comptabilité de la norme litigieuse avec, à la fois, la Constitution et les traités internationaux.

[Énoncé 2 après] À propos des normes infra-légales, tels que les règlements et arrêtés grand-ducaux et ministériels, le juge ordinaire veille non seulement à la légalité d'une telle norme mais aussi à ce qu'elle soit, à la fois, conforme à la Constitution comme aux traités internationaux. [1] Il dispose donc d'une compétence globale au regard du contrôle des normes infra-légales.

- l'ajout d'un connecteur logique :

[Énoncé 4 avant] Il est obligé de saisir la Cour constitutionnelle par voie de question préjudicielle et, par la suite, de se conformer à la décision rendue par celle-ci.

[Énoncé 4 après] En revanche, au cas où il se défie de la constitutionnalité de la même loi, il est obligé de saisir la Cour constitutionnelle par voie de question préjudicielle et, par la suite, de se conformer à la décision rendue par celle-ci.

[Énoncé 3 avant] Elle est perçue comme une surface de projection et un processus de représentations multiples qui sont créés par des acteurs sociaux (p. ex. les anciens résistants) et leurs médias (p.ex. les monuments et musées) après la guerre.

[Énoncé 3 après] Elle est, au contraire, perçue comme une surface de projection et un processus de représentations multiples qui sont créés par des acteurs sociaux (p. ex. les anciens résistants) et leurs médias (p. ex. les monuments et musées) après la guerre.

- le changement de sujet (répartition thème/rhème inversée).

Le passage de la phrase impersonnelle à la phrase passive engendre un effacement du complément d'agent et la disparition de la prise en charge énonciative comme marqueur, comme le montrent les exemples ci-dessous :

[Énoncé 5 avant] Du côté musulman, il s'agit d'une condition sine qua non de la reconnaissance car sollicitée par les États (Maréchal, 2001).

[Énoncé 5 après] La constitution de ces organes représentatifs est très souvent ressentie comme une condition sine qua non de la reconnaissance car sollicitée par les États (Maréchal, 2001).

[Énoncé 1 avant] Les sages-femmes admises selon les anciennes formes sont recensées dans un document préfectoral daté de 1807.

[Énoncé 1 après] Un document préfectoral daté de 1807 recense les sages-femmes admises selon les anciennes formes.

Nous n'avons pas abordé la thématique de la syntaxe directement en cours, mais les changements de sujet montrent que les doctorants ressentent le besoin de proposer une nouvelle prise en charge énonciative lors de la révision.

\subsubsection{La deuxième modification : au niveau du paragraphe}

La modification au niveau du paragraphe est repérable par :

- la réorganisation des énoncés entre eux (10\%), notamment en en supprimant un, voire plusieurs : 
"À la fin j'ai éliminé pas mal de phrases et changé presque toutes les phrases »

- la fusion de deux énoncés en un $(6 \%)$ :

[Énoncé 10 avant] Il est dès lors important de ne pas sous-estimer les conséquences fiscales et sociales d'une activité salariée au Grand-Duché de Luxembourg.

[Énoncé 11 avant] (P10') Elles varieront bien entendu selon la situation personnelle et professionnelle du salarié. Elles méritent tout de même que l'on s'y attarde pour limiter les coûts supplémentaires liés à d'éventuels redressements aussi bien dans le chef du salarié que dans celui de l'employeur

[Énoncé 10 et 11 après] Concrètement, les impacts fiscaux et sociaux pour les travailleurs frontaliers occupés au Luxembourg vont essentiellement dépendre de la situation personnelle et professionnelle de chaque salarié. Une analyse, au cas par cas, est ainsi fortement recommandée pour éviter toutes complications administratives et coûts inutiles pour le salarié comme pour l'employeur.

- la division d'un énoncé en plusieurs (19\%) :

[Énoncé 4 avant] Alors que les comptes des officiers de justice des XIV et $\mathrm{XV}^{\mathrm{e}}$ siècles ne mentionnent aucunement des injures et que, de nos jours, les injures ne sont plus que rarement portées devant la justice, elles étaient pour les $\mathrm{XVI}^{\mathrm{e}}$ et $\mathrm{XVII}^{\mathrm{e}}$ siècles «une des plus fréquentes expressions de la violence [et] à l'origine de nombre de rixes qui [pouvaient] dégénérer en coups et blessures graves, voire provoquer la mort. »

[Énoncé 4 après] Les comptes des officiers de justice des $\mathrm{XIV}^{\mathrm{e}}$ et $\mathrm{XV}^{\mathrm{e}}$ siècles ne mentionnent aucunement des injures, néanmoins nous retrouvons une criminalisation de la parole dans les deux siècles suivants.

[Énoncé 5 après] Alors que, de nos jours, les injures ne sont - à nouveau - plus que rarement portées devant la justice, elles étaient pour les périodes en question « une des plus fréquentes expressions de la violence [et] à l'origine de nombre de rixes qui [pouvaient] dégénérer en coups et blessures graves, voire provoquer la mort. »

Cette opération est réalisée dans un tiers des changements apportés. Les doctorants voient le travail sur leur texte passer par une syntaxe plus précise pour réorganiser les idées du paragraphe. Ils travaillent plus volontiers à un niveau micro, car ils font accéder le lecteur au sens par le détail qui se cache dans les rouages de l'énoncé.

\subsubsection{La troisième modification : la métaréflexion}

41 Nous avons relevé des commentaires métadiscursifs ( $17 \%$ des modifications) dans les secondes versions du paragraphe portant sur la ponctuation, sur la répétition, sur l'anaphore, sur l'arrimage et sur le connecteur.

"J'ai n'ai pas repris une seule phrase de l'ancien paragraphe, ça me perturbait d'essayer de réutiliser. »

«Je me permets de mettre les trois paragraphes, car les deux derniers font en fait plus ou moins partie de la même idée. »

«J'ai scindé les phrases en deux et me suis assurée que les idées ne se mélangent plus. Mon but était de faire en sorte que la phrase suivante complète la précédente, ou, à tout le moins, que les idées se suivent logiquement. »

«J'ai découpé différemment les phrases, pour rendre le texte plus clair, plus fluide. Ainsi, le paragraphe a maintenant six phrases au lieu de seulement trois phrases. J'ai changé aussi l'ordre des mots dans certaines d'entre elles et j'ai remplacé une anaphore par une autre. »

42 Les doctorants recréent, remodèlent, repensent leur texte. Ils manipulent la phrase comme un outil pour mieux véhiculer leur message. Ils le font d'eux-mêmes puisque notre partie théorique a davantage traité de la prise de conscience du message à délivrer 
au lecteur que du métalangage grammatical. Ils reviennent à la notion de phrase et de syntaxe et ne parlent plus directement du contenu.

Ce retour d'expérience est également positif dans les questionnaires qu'ils ont remplis à la fin du cours. À la question "Concernant votre processus d'écriture, avez-vous remarqué que votre manière d'aborder des lectures de textes a évolué au fil des séances?», $80 \%$ des doctorants répondent par l'affirmative. Les commentaires ${ }^{3}$ cidessous montrent la prise de conscience sur ce qu'apportent la réécriture et la sensibilisation à la cohérence textuelle :

«Meilleure visibilité de la structure d'un texte"

«Lecture plus organisée, recherchant en premier les points clé et l'articulation des idées dans le texte "

«[...] de manière générale je suis plus attentive concernant la structure et la cohérence des textes que je lis. »

" Plus d'attention aux détails et à la cohérence textuelle »

Néanmoins, un doctorant fait part de sa retenue :

«Trop abstrait et distant de ma façon d'écrire et de réfléchir à mon processus de rédaction. »

17 doctorants sur les 18 qui ont participé à ce cours considèrent avoir posé un nouveau regard sur leur façon de rédiger et estiment dorénavant savoir réfléchir pour rendre leurs textes plus cohérents et plus compréhensibles.

Comme les résultats des commentaires le montrent, les participants se sont particulièrement concentrés sur l'usage des connecteurs et, en réponse à la question du thème qu'ils aimeraient approfondir à l'avenir, seul un doctorant a choisi l'utilisation implicite et explicite des connecteurs logiques. 17 des 18 doctorants souhaitent une intervention complémentaire sur l'arrimage informatif qui consiste en une hiérarchisation des informations dans la phrase. Ils sont en demande de plus d'accompagnement pour aiguiser leur habileté à communiquer un message et à l'élaborer lors des phases de réécriture.

\section{Conclusion}

Bien que la question du lecteur-récepteur soit travaillée dans certains cursus scolaires en prenant en compte les révisions de textes en adéquation avec leur destinataire, il semble qu'écrire en se positionnant également comme lecteur soit une réflexion qui n'ait pas été effectuée avant le cours et qui interpelle les doctorants. Nous avons (ré-)activé le concept de cohérence textuelle auprès des doctorants, suite à deux semestres d'atelier d'écriture intégrant la relecture entre pairs. Cette activité sur la cohérence textuelle est venue s'inscrire dans la continuité d'une réflexion visant à répondre à des constats récurrents de paragraphes "peu clairs » et "phrases bancales » conformément aux commentaires formulés antérieurement. À l'avenir, il serait intéressant d'analyser également les commentaires évaluatifs de type "peu clair», "très/peu fluide", «mal dit", « maladroit », etc., qui sont de l'ordre de l'impression générale plutôt que du métalangage grammatical (Charolles, 1978).

47 Parfaire leur texte pour faciliter l'interprétation du lecteur et donner l'occasion aux participants de travailler sur un extrait de texte décontextualisé a naturellement amené les doctorants à s'interroger sur la syntaxe et les liens entre les énoncés. 

pour lesquelles on écrit, les idées s'affinent, le texte devient plus cohérent et le questionnement sur ses propres réflexions se fait plus complexe. Les paragraphes que nous avons demandé aux doctorants de réécrire à l'issue du cours manifestent que l'idée d'intéresser le lecteur, de lui faire suivre son cheminement propre est intégrée à l'écriture.

49

En termes de grammaticalité, nous n'avons pas constaté de différence notable entre le premier texte apporté en cours et sa nouvelle version, puisque les deux versions $d u$ paragraphe étaient «correctes », mais en termes d'acceptabilité, le remaniement de la syntaxe a permis de mieux véhiculer l'idée du paragraphe et de mieux « arrimer » chaque énoncé au contexte.

50 Enfin, pour répondre à notre dernière question sur la possible différence de résultats entre les doctorants en sciences humaines et en droit, nous n'avons pas noté de différence particulière dans le traitement du texte proprement dit, mais, en revanche, dans la réception de l'approche. Comme nous l'avons expliqué, les deux groupes ont vécu deux mises en route distinctes. Les participants du groupe de sciences humaines ont eu des difficultés à réaliser les exercices à partir des textes extraits de Scientext. En revanche, les doctorants en droit ont immédiatement cerné l'opérationnalité des concepts de cohérence et de pertinence grâce à des exercices fondés sur des extraits de leurs propres travaux.

51 Les résultats font apparaitre que le lien entre la grammaire et la cohérence textuelle n'est pas effectué consciemment par les doctorants. La correction grammaticale n'est donc pas directement en cause, mais une prise de conscience des choix syntaxiques aide à la construction de la pensée.

52 Comme les doctorants sont en demande d'approfondissement de la question de l'arrimage grâce aux connecteurs, nous pourrions envisager la suite de ce cours en salle informatique. L'activité pourrait consister à sélectionner un connecteur dans Scientext et à commenter l'acceptabilité des paragraphes qui l'intègrent.

\section{BIBLIOGRAPHIE}

BECKER, Howard. (1986). Écrire les sciences sociales. Commencer et terminer son article, sa thèse ou son livre. Paris : Economica.

CECRL - Cadre européen commun de référence pour les langues. (2000). Strasbourg : Conseil de l'Europe / Paris : Didier.

CAVAlla, Cristelle \& LoISEAU, Mathieu. (2013). Scientext comme corpus pour l'enseignement. Dans A. Tutin \& F. Grossmann (dir.), L'écrit scientifique : du lexique au discours. Autour de Scientext (p. 163-182). Rennes : Presses universitaires de Rennes. 
CHAMBERS, Angela. (2007). Popularising Corpus Consultation by Language Learners and Teachers. Dans E. Hildalgo, L. Quereda \& J. Santana (dir.), Corpora in the Foreign Language Classroom (p. 3-16). Leiden : Brill.

CHAROLLES, Michel. (1978). Introduction aux problèmes de la cohérence des textes. Langue française, 38, 7-42.

CHO, Kwangsu, \& MACARThuR, Charles. (2010). Student Revision with Peer and Expert Reviewing. Learning and Instruction, 20, 328-338.

Сномsку, Noam. (1965). Aspects of the Theory of Syntax. Cambridge, MA : MIT Press.

Cote, Robert Arthur. (2013). The Role of Student Attitude towards Peer Review in Anonymous Electronic Peer Review in an EFL Writing Classroom. Tucson : University of Arizona.

CUQ, Jean-Pierre. (1996). Une introduction à la didactique de la grammaire en français langue étrangère. Paris : Didier/Hatier.

De BeAugrande, Robert \& DRESSLER, Wolfgang. (1981). Introduction to Text Linguistics. Londres : Longman.

DE SCHEPPER, Catherine. (2010). Acculturation aux discours universitaires. Poser les variables de l'intervention didactique. Diptyque, 18, 93-126.

DonahuE, Christiane. (2008). Écrire à l'université. Analyse comparée en France et aux États-Unis. Villeneuve-d'Ascq : Presses universitaires du Septentrion.

GAGNON, Odette. (1998). Manifestations de la cohérence et de l'incohérence dans des textes argumentatifs d'étudiants universitaires québécois. Québec : Université Laval.

GAGNON, Odette. (2003). Apprécier la cohérence d'un texte : l'arrimage des énoncés. Québec français, 128, 62-66.

GaGnON, Odette. (2015). Travailler la cohérence du texte. Dans F. Boch \& C. Frier (dir.), Écrire dans l'enseignement supérieur : des apports de la recherche aux outils pédagogiques (p. 100-135). Grenoble : ELLUG.

Goody, Jack. (1979). La raison graphique : la domestication de la pensée sauvage. Paris : Minuit. HALLIDAY, Michael A. K. \& HASAN, Ruqaiya. (1976). Cohesion in English. Londres : Longman. HоввS, Jerry. (1983 [1978]). Why Is Discourse Coherent. Dans F. Neubauer (dir.), Coherence in Natural Language Texts (p. 29-71). Hambourg : Buske.

LABORDE-MilAA, Isabelle, Boch, Françoise \& REUTER, Yves (dir.). (2004). Les écrits universitaires. Pratiques, 121-122.

Min, Hui-Tzu. (2006). The Effects of Trained Peer Review on EFL Students' Revision Types and Writing Quality. Journal of Second Language Writing, 15(2), 118-141.

MoESCHLER, Jacques \& ReBoul, Anne. (1994). Dictionnaire encyclopédique de pragmatique. Paris : Seuil. Pollet, Marie-Christine. (2004). Appropriation et écriture de savoirs chez des étudiants de première année. Pratiques, 121-122, 81-94.

SPERBER, Dan \& WiLSON, Deirdre. (1989). La Pertinence : communication et cognition. Paris : Minuit. VAN DIJK, Teun Adrianus \& KINTSCH, Walter. (1983). Strategies of Discourse Comprehension. New York : Academic Press. 


\section{NOTES}

1. Les doctorants soit ont le français comme langue maternelle, soit maitrisent cette langue à un niveau $\mathrm{C} 1$ (prérequis pour participer au cours).

2. Nous présenterons des exemples d'altérations en 3.3.

3. Ces commentaires correspondent quasiment à la totalité des commentaires puisque tous les doctorants n'ont pas annoté les évaluations.

\section{RÉSUMÉS}

Cet article a pour objet de créer un pont entre la grammaire de la phrase et la cohérence textuelle dans le processus d'écriture des doctorants. Il s'appuie sur une étude de cas menée auprès de deux groupes : les doctorants de sciences humaines d'un côté et ceux de droit de l'autre. Le corpus de cette recherche se compose de paragraphes écrits et retravaillés par les doctorants eux-mêmes dans le cadre d'un atelier d'écriture. La question-clé qui oriente la recherche est de savoir si l'approche par la grammaire de la phrase a une répercussion sur la qualité des écrits des doctorants. Les résultats de réécriture de paragraphes font apparaitre trois types de modifications. La principale modification se fait au sein même de l'énoncé et non du paragraphe. La seconde modification consiste en la réorganisation des énoncés au sein du paragraphe. Enfin, la troisième modification réalisée par les doctorants sur leur paragraphe est l'ajout d'un commentaire méta-réflexif indiquant leurs démarches. Par ailleurs, les deux versions $\mathrm{du}$ paragraphe sont grammaticalement correctes, la correction grammaticale n'est donc pas directement en cause, mais une bonne maitrise de celle-ci aide à la construction de la pensée.

The objective of this article is to build a bridge between sentence grammar and textual coherence within the writing process of doctoral students. It draws upon a study conducted with two groups: doctoral students in the humanities on the one hand and doctoral students in law on the other hand. The body of this research consists of paragraphs written and redrafted by the doctoral students themselves in the context of a writing workshop. The key research question is to ascertain whether a grammar-based approach of the sentence has a repercussion on the quality of the writings produced by doctoral students. The results of the redrafting of the paragraphs reveal three types of changes. The main change occurs within the actual utterance rather than within the paragraph. The second change consists in reorganising the utterances within the paragraph. And finally, the third most frequent action performed at paragraph level by doctoral students is to add a meta-reflexive comment clarifying their approach. Both versions of the paragraph are incidentally grammatically correct. Grammatical correctness thus does not have a direct relevance, but a good command of grammar helps in the construction of thought. 
INDEX

Keywords : textual coherence, academic literacy, paragraph, utterance, doctoral students, grammaticality

Mots-clés : cohérence textuelle, littéracie universitaire, paragraphe, énoncé, doctorants, grammaticalité

\section{AUTEUR}

\section{ÈVE LEJOT}

Université du Luxembourg, Unité de recherche Identités. Politiques, Sociétés, Espaces (IPSE) 